\title{
Fractal Dimension for the Nonautonomous Stochastic Fifth-Order Swift-Hohenberg Equation
}

\author{
Yanfeng Guo $\mathbb{D}^{1,2}$ Chunxiao Guo ${ }^{1}{ }^{3}$ and Yongping $\mathrm{Xi}^{3}$ \\ ${ }^{1}$ School of Mathematics and Physics, China University of Geosciences, Wuhan 430074, Hubei, China \\ ${ }^{2}$ School of Science, Guangxi University of Science and Technology, Liuzhou 545006, Guangxi, China \\ ${ }^{3}$ School of Science, China University of Mining and Technology, Beijing 100083, China \\ Correspondence should be addressed to Chunxiao Guo; guochunxiao1983@sina.com
}

Received 13 August 2020; Revised 27 August 2020; Accepted 5 September 2020; Published 16 September 2020

Academic Editor: Karthikeyan Rajagopal

Copyright ( 2020 Yanfeng Guo et al. This is an open access article distributed under the Creative Commons Attribution License, which permits unrestricted use, distribution, and reproduction in any medium, provided the original work is properly cited.

Some dynamics behaviors for the nonautonomous stochastic fifth-order Swift-Hohenberg equation with additive white noise are considered. The existence of pullback random attractors for the nonautonomous stochastic fifth-order Swift-Hohenberg equation with some properties is mainly investigated on the bounded domain and unbounded domain, through the Ornstein-Uhlenbeck transformation and tail-term estimates. Furthermore, on the basis of some conditions, the finiteness of fractal dimension of random attractor is proved.

\section{Introduction}

Swift and Hohenberg proposed the Swift-Hohenberg (S-H) equation as a model for the convective instability in the Rayleigh-Bénard convection in 1997 [1]. There have been some results for the classical S-H equation [2-6]. Peletier and his collaborators have studied the S-H equation from different aspects, such as the stability of stationary solutions and pattern selections of solutions [7-9]. Recently, some results about pullback attractor $[10,11]$ and uniform attractor [12] of S-H equation are investigated. As we know, more and more authors investigated the random attractors and have obtained many important results. Meanwhile, there has been tremendous interest in developing the fractal dimension estimate of random attractors in recent years (see [13-19] and the references there in).

We consider the stochastic fifth-order S-H equation driven by additive white noise:

$$
\begin{aligned}
\mathrm{d} u & +\left(\Delta^{2} u+2 \Delta u+a u+u^{5}-g(x, t)\right) \mathrm{d} t \\
& =\phi(x) \mathrm{d} W(t), \quad((x, t) \in D \times(\kappa,+\infty), \kappa \in \mathbb{R},
\end{aligned}
$$

with boundary condition

$$
u(t, x)=0, \quad x \in \partial D
$$

and the initial condition

$$
u(x, \kappa)=u_{\kappa}(x), \quad x \in D,
$$

where $a>0$ and $D \subset \mathbb{R}^{2}$ is a bounded smooth domain.

There are some results about dynamics behaviors for the classical autonomous stochastic S-H equation [20, 21]. Except for the study of the existence of the random attractor [22-24], Zhou et al. have established an efficient theory about the finite fractal dimensions of random attractor $[25,26]$. To our knowledge, the fractal dimension estimate has been barely studied for the random attractors of the stochastic fifth-order S-H equation yet. According to the ideas in [21, 25-28], stochastic dynamics behaviors of the random attractor are considered for stochastic equations (1)-(3) in two cases.

Firstly, we mainly give the existence of random attractor for the fifth-order S-H equation corresponding to (1)-(3). A few results about the dynamics behaviors of the fifth-order S-H equation with additive noise have been given when the nonlinear term is five ordered. Due to the increasing order, more difficult terms can be produced to derive uniform estimates for the solution of equations 
(1)-(3). In order to overcome these difficulties aroused by the fifth-order term, we mainly use the method of integration by parts after Ornstein-Uhlenbeck transformation. Notice that the uniform estimates are independent of bounded region $D$, and we can obtain the existence of random attractor by proving that the random dynamical system is asymptotically compact through tail-term estimates on unbounded domain $\mathbb{R}^{2}$.

Secondly, we are devoted to the finiteness of fractal dimension for the random attractor of (1)-(3). Because of the complexity of proving the boundedness of fractal dimension on unbounded domain, we pay attention to studying the fifth-order S-H equation on bounded domain $D$. Furthermore, we will discuss the case of unbounded domain. Here, in order to obtain the boundedness of the fractal dimension, some sufficient conditions are proposed [16-18].

\section{Preliminaries}

We give the theorem related to random attractors. Since these conclusions are classic, we will not discuss them in detail, and readers can check the relevant literature [22-24, 29, 30].

In this paper, we will use $\|\cdot\|$ to denote the norm and $(\cdot, \cdot)$ to denote the inner product in $L^{2}\left(\mathbb{R}^{2}\right)$ or $L^{2}(D)$, where $D$ is a bounded smooth domain. In the case of bounded domain, for simplicity, we use the notation $H$ to present the space $L^{2}(D)$. We will write the norm of $L^{p}\left(\mathbb{R}^{2}\right)\left(L^{p}(D)\right)$ as $\|\cdot\|_{L^{p}}$ and use $\|\cdot\|_{X}$ to denote the norm of Banach space $X$ and $\|\cdot\|_{s}$ to denote the norm in $H^{s}(D)$ or $H^{s}\left(\mathbb{R}^{2}\right)$.

The continuous random dynamical system will be showed for the stochastic fifth-order $\mathrm{S}-\mathrm{H}$ equation on $L^{2}\left(\mathbb{R}^{2}\right)\left(\right.$ or $\left.L^{2}(D)\right)$ :

$$
\mathrm{d} u+\left(\Delta^{2} u+2 \Delta u+a u+u^{5}-g(x, t)\right) \mathrm{d} t=\sum_{i=1}^{m} \phi_{i}(x) \mathrm{d} W_{i}(t)
$$

with boundary condition

$$
u(t, x)=0, \quad x \in \partial D,
$$

and with the initial condition

$$
u(x, \kappa)=u_{\kappa}(x), x \in D,
$$

where $\phi_{i}(x) \in H^{4}\left(\mathbb{R}^{2}\right)(i=1, \ldots, m)$ is the smooth enough function. $W$ is a two-sided real-value Wiener process on a probability space and $g(x, \cdot) \in C_{b}(\mathbb{R}, H)$ is given.

Let $\widetilde{z}(\omega)=-a \int_{-\infty}^{0} e^{a \kappa} \omega(\kappa) \mathrm{d} \kappa, \omega \in \Omega$, which is a unique stationary solution of the equation [29]:

$$
\mathrm{d} \widetilde{z}+a \widetilde{z} \mathrm{~d} t=\mathrm{d} W .
$$

In addition, for each fixed $\omega \in \Omega, \widetilde{z}\left(\theta_{t} \omega\right)$ is pathwise continuous. And there is a tempered function $r(\omega)>0$ :

$$
\left|\widetilde{z}\left(\theta_{t} \omega\right)\right|^{2}+\left|\widetilde{z}\left(\theta_{t} \omega\right)\right|^{p} \leq e^{a-6 / 2|t|} r(\omega), \quad t \in \mathbb{R}, \mathrm{P}-\text { a.e. } \omega \in \Omega .
$$

Given a translation $\quad v\left(t, \kappa, \omega, v_{\kappa}\right)=u$ $\left(t, \kappa, \omega, u_{\kappa}\right)-z\left(\theta_{t} \omega\right)$, one yields $v_{\kappa}=u_{\kappa}-z(\omega)$, where $z\left(\theta_{t} \omega\right)=\phi(x) \widetilde{z}\left(\theta_{t} \omega\right)$. Then, we obtain

$$
\begin{aligned}
& \frac{\mathrm{d} v}{\mathrm{~d} t}+\Delta^{2} v+2 \Delta v+2 \Delta z\left(\theta_{t} \omega\right)+a v+\Delta^{2} z\left(\theta_{t} \omega\right)+v^{5} \\
& \quad+z^{5}\left(\theta_{t} \omega\right)+5 v^{4} z\left(\theta_{t} \omega\right)+10 v^{3} z^{2}\left(\theta_{t} \omega\right)+10 v^{2} z^{3}\left(\theta_{t} \omega\right) \\
& \quad+5 v z^{4}\left(\theta_{t} \omega\right)=g(x, t) \\
& v(t, x)=0, \quad x \in \partial D \\
& v(\kappa, \kappa, \omega, x)=v_{\kappa}(\omega)=u_{\kappa}(x)-z(\omega)
\end{aligned}
$$

By the Galerkin method, for all $v_{\kappa} \in H$, as proved in [31], one can show that systems (9)-(11) are well-posed for every $\omega \in \Omega$ in $H$. A continuous cocycle $\Phi: \mathbb{R}^{+} \times \mathbb{R} \times \Omega \times$ $H \longrightarrow H$ is defined by

$$
\Phi\left(t, \kappa, \omega, u_{\kappa}\right)=\Phi(t, \kappa, \omega) u_{\kappa}=u\left(t+\kappa, \kappa, \theta_{-\kappa} \omega, u_{\kappa}\right),
$$

and a cocycle $\Psi: \mathbb{R}^{+} \times \mathbb{R} \times \Omega \times H \longrightarrow H$ by

$$
\Psi\left(t, \kappa, \omega, v_{\kappa}\right)=\Psi(t, \kappa, \omega) v_{\kappa}=v\left(t+\kappa, \kappa, \theta_{-\kappa} \omega, v_{\kappa}\right),
$$

where $v_{\kappa}=u_{\kappa}-z(\omega)$. Notice that the continuous dynamical systems $\Phi$ and $\Psi$ are equivalent. Similarly, $\Phi: \mathbb{R}^{+} \times \mathbb{R} \times \Omega \times$ $L^{2}\left(\mathbb{R}^{2}\right) \longrightarrow L^{2}\left(\mathbb{R}^{2}\right)$ is a continuous cocycle.

Let $B=\{B(\kappa, \omega): \kappa \in \mathbb{R}, \omega \in \Omega\}$, which are a family of bound nonempty subsets of $H$ (or $L^{2}\left(\mathbb{R}^{2}\right)$ ) and

$$
\lim _{t \longrightarrow+\infty} e^{-c t}\left\|B\left(\kappa+t, \theta_{t} \omega\right)\right\|^{2}=0, \quad \forall c>0, \kappa \in \mathbb{R}, \omega \in \Omega,
$$

where $\|B\|=\sup _{u \in B}\|u\|$. Here, we always assume that $\mathscr{D}$ is the collection of all tempered families of nonempty subsets of $H$. Furthermore, we prove that there exist $\mathscr{D}$-pullback attractors for cocycle $\Phi$. In the whole paper, we assume $g(x, \cdot) \in C_{b}(\mathbb{R}, H)$ with

$$
\|g\|^{2}=\sup _{t \in \mathbb{R}}\|g(\cdot, t)\|^{2}<+\infty .
$$

\section{Some Uniform Estimates}

The uniform estimates are derived for solutions of systems (9)-(11) on bounded domain $D$ and $\mathbb{R}^{2}$, respectively.

Lemma 1. Suppose $a>6$ and (8) holds. Then, for every $\kappa \in \mathbb{R}, \omega \in \Omega$, and $B=\{B(\kappa, \omega): \kappa \in \mathbb{R}, \omega \in \Omega\} \in \mathscr{D}$, there is a $T_{0}(\kappa, \omega, B) \geq 1$ satisfying

$$
\begin{gathered}
\left\|v\left(\kappa, \kappa-t, \theta_{-\kappa} \omega, v_{\kappa-t}\right)\right\|^{2} \leq r_{0}(\kappa, w), \\
\left\|u\left(\kappa, \kappa-t, \theta_{-\kappa} \omega, u_{\kappa-t}\right)\right\|^{2} \leq r_{1}(\kappa, w),
\end{gathered}
$$

for $\forall t \geq T_{0}(\kappa, \omega, B)$, where $v$ is the solution of systems (9)-(11), $v_{\kappa-t}+z\left(\theta_{-t} \omega\right) \in B\left(\kappa-t, \theta_{-t} \omega\right)$, and $r_{0}(\kappa, w)$ and $r_{1}(\kappa, w)$ are two tempered random variables. 
Proof. From (9), we deduce

$$
\begin{aligned}
\frac{1}{2} \frac{\mathrm{d}}{\mathrm{d} t}\|v\|^{2}+\|\Delta v\|^{2}= & -a\|v\|^{2}-\|v\|_{L^{6}}^{6} \\
& -\left(2 \Delta v+2 \Delta z\left(\theta_{t} \omega\right)+\Delta^{2} z\left(\theta_{t} \omega\right), v\right) \\
& -\left(z^{5}\left(\theta_{t} \omega\right)+5 v^{4} z\left(\theta_{t} \omega\right)+10 v^{3} z^{2}\left(\theta_{t} \omega\right), v\right) \\
& -5\left(2 v^{2} z^{3}\left(\theta_{t} \omega\right)+v z^{4}\left(\theta_{t} \omega\right), v\right)+(g(t), v) .
\end{aligned}
$$

Since

$$
\begin{aligned}
\left(10 v^{3} z^{2}\left(\theta_{t} \omega\right), v\right)= & 10 \int_{D} v^{4} z^{2}\left(\theta_{t} \omega\right) \mathrm{d} x \geq 0,\left(5 v z^{4}\left(\theta_{t} \omega\right), v\right) \\
& =5 \int_{D} v^{2} z^{4}\left(\theta_{t} \omega\right) \mathrm{d} x \geq 0
\end{aligned}
$$

applying the Hölder inequality and the $\varepsilon$-Young inequality, one gets

$$
\begin{aligned}
\frac{\mathrm{d}}{\mathrm{d} t}\|v\|^{2}+\|v\|_{L^{6}}^{6}+(a-6)\|v\|^{2}+\|\Delta v\|^{2} \leq & C\left(\left|\tilde{z}\left(\theta_{t} \omega\right)\right|^{10}\right. \\
& \left.+\left|\widetilde{z}\left(\theta_{t} \omega\right)\right|^{6}+\left|\widetilde{z}\left(\theta_{t} \omega\right)\right|^{2}\right) \\
& +\frac{1}{a}\|g(t)\|^{2} \doteq F\left(\theta_{t} \omega\right) \\
& +\frac{1}{a}\|g(t)\|^{2},
\end{aligned}
$$

when $r \geq \kappa-t$, by the Gronwall inequality on $[\kappa-t, r]$ and substituting $\theta_{-\kappa} \omega$ for $\omega$, one has

$$
\begin{aligned}
& \left\|v\left(r, \kappa-t, \theta_{-\kappa} \omega, v_{\kappa-t}\right)\right\|^{2} \leq e^{-(a-6)(r+t-\kappa)}\left\|v_{\kappa-t}\right\|^{2} \\
& +\int_{\kappa-t}^{r}\left(e^{(6-a)(r-s)} F\left(\theta_{s-\kappa} \omega\right)+\frac{e^{(6-a)(r-s)}}{a}\|g(s)\|^{2}\right) \mathrm{d} s \\
& \leq e^{-(a-6) t}\left\|v_{\kappa-t}\right\|^{2}+\int_{-\infty}^{0}\left(e^{-(6-a) s} F\left(\theta_{s} \omega\right)\right. \\
& \left.\quad+\frac{e^{-(6-a) s}}{a}(g(s+\kappa)]^{2}\right) \mathrm{d} s .
\end{aligned}
$$

From (20), since $v_{\kappa-t}+z\left(\theta_{-t} \omega\right) \in B\left(\kappa-t, \theta_{-t} \omega\right)$, and $B=$ $\{B(\kappa, \omega): \kappa \in \mathbb{R}, \omega \in \Omega\}$ is tempered, and there is $T_{0}=T_{0}(\kappa, \omega, B)>1$ satisfying

$$
e^{-(a-6) t}\left\|v_{\kappa-t}\right\|^{2} \leq 1, \forall t \geq T_{0}
$$

From (20), we can deduce

$$
\begin{aligned}
& \| v\left(\kappa, \kappa-t, \theta_{-\kappa} \omega, v_{\kappa-t} \|^{2}\right. \\
& \quad \leq 1+C \int_{-\infty}^{0} e^{\frac{(a-6) s}{2}} r(\omega) \mathrm{d} s+\frac{1}{a} \int_{-\infty}^{0}\|\|(s+\kappa) \|^{2} e^{(a-6) s} \mathrm{~d} s \\
& \quad \leq e^{-(a-6) t}\left\|v_{\kappa-t}\right\|^{2}+\frac{2 C}{a-6} r(\omega)+\frac{1}{a} \int_{-\infty}^{0} e^{(a-6) s}\|g(s+\kappa)\|^{2} \mathrm{~d} s \\
& \quad r_{0}(\kappa, \omega), \forall t \\
& \geq T_{0}(\kappa, \omega, B) .
\end{aligned}
$$

For all $v_{\kappa-t}+z\left(\theta_{-t} \omega\right) \in B\left(\kappa-t, \theta_{-t} \omega\right)$, we have

$$
\begin{aligned}
\left\|u\left(\kappa, \kappa-t, \theta_{-\kappa} \omega, u_{\kappa-t}\right)\right\|^{2} \leq & 2\|z(\omega)\|^{2}+2 \\
& +C\left(\int_{-\infty}^{0} e^{(a-6) s / 2} r(\omega) \mathrm{d} s\right. \\
& \left.+\int_{-\infty}^{0}\|g(s+\kappa)\|^{2} e^{(a-6) s} \mathrm{~d} s\right) \\
\doteq & r_{1}(\kappa, \omega), \forall \kappa \in \mathbb{R}, \omega \in \Omega .
\end{aligned}
$$

Denote

$K(\kappa, \omega)=\left\{u \in H:\|u\|^{2} \leq r_{1}(\kappa, \omega), \omega \in \Omega \kappa \in \mathbb{R}\right\} \in \mathscr{D}$.

Then, $K(\kappa, \omega)$ is an absorbing set for $\Phi$ in $\mathscr{D}$

Remark 1. Using the Gronwall lemma, we can obtain the following results. For every $\kappa \in \mathbb{R}, \omega \in \Omega$, and $B=\{B(\kappa, \omega): \kappa \in \mathbb{R}, \omega \in \Omega\} \in \mathscr{D}$ $T_{1}(\kappa, \omega, B) \geq T_{0} \geq 1$ satisfying

$$
\begin{aligned}
& \int_{\kappa-1}^{\kappa}\left\|v\left(s, \kappa-t, \theta_{-\kappa} \omega, v_{\kappa-t}\right)\right\|^{2} \mathrm{~d} s \leq r_{2}(\kappa, w), \forall t \geq T_{1}(\kappa, \omega, B), \\
& \int_{-t}^{0} e^{(a-6) s}\left\|\Delta v\left(s+\kappa, \kappa-t, \theta_{-\kappa} \omega, v_{\kappa-t}\right)\right\|^{2} \mathrm{~d} s \\
& \quad+\int_{-t}^{0} e^{(a-6) s}\left\|v\left(s+\kappa, \kappa-t, \theta_{-\kappa} \omega, v_{\kappa-t}\right)\right\|_{L^{6}}^{6} \mathrm{~d} s \\
& \quad \leq r_{3}(\kappa, \omega), \forall t \geq T_{1}(\kappa, \omega, B),
\end{aligned}
$$

where $v$ is the solution of systems (9)-(11), $v_{\kappa-t}+z\left(\theta_{-t} \omega\right) \in B\left(\kappa-t, \theta_{-t} \omega\right)$, and $r_{2}(\kappa, w)$ and $r_{2}(\kappa, w)$ are tempered random variables.

Lemma 2. Suppose $a>6$ and (15) holds. Then, for every $\kappa \in \mathbb{R}, \omega \in \Omega$, and $B=\{B(\kappa, \omega): \kappa \in \mathbb{R}, \omega \in \Omega\} \in \mathscr{D}$, there is a $T_{1}(\kappa, \omega, B) \geq T_{0} \geq 1$ satisfying

$$
\left\|\Delta v\left(\kappa, \kappa-t, \theta_{-\kappa} \omega, v_{\kappa-t}\right)\right\|^{2} \leq r_{4}(\kappa, \omega), \forall t \geq T_{1}(\kappa, \omega, B),
$$

where $v$ is the solution of systems (9)-(11), $v_{\kappa-t}+z\left(\theta_{-t} \omega\right) \in B\left(\kappa-t, \theta_{-t} \omega\right)$, and $r_{4}(\kappa, w)$ and $r_{5}(\kappa, w)$ are tempered random variables.

Proof. By (9), one obtains

$$
\begin{aligned}
& \frac{1}{2} \frac{\mathrm{d}}{\mathrm{d} t}\|\Delta v\|^{2}+\left(2 \Delta v, \Delta^{2} v\right)+\left(2 \Delta z\left(\theta_{t} \omega\right)+\Delta^{2} z\left(\theta_{t} \omega\right), \Delta^{2} v\right) \\
& \quad+a\|\Delta v\|^{2}+\|\Delta v\|^{2}+\left(v^{5}, \Delta^{2} v\right)+\left(z^{5}\left(\theta_{t} \omega\right), \Delta^{2} v\right) \\
& \quad+\left(\left(v^{4} z\left(\theta_{t} \omega\right), \Delta^{2} v\right)+\left(10 v^{3} z^{2}\left(\theta_{t} \omega\right), \Delta^{2} v\right)\right. \\
& \quad+\left(10 v^{2} z^{3}\left(\theta_{t} \omega\right), \Delta^{2} v\right)+\left(5 v z^{4}\left(\theta_{t} \omega\right) \Delta^{2} v\right)=\left(g(x, t), \Delta^{2} v\right) .
\end{aligned}
$$

It is easy to get 


$$
\left|2\left(\Delta v, \Delta^{2} v\right)\right| \leq \frac{1}{4}\left\|\Delta^{2} v\right\|^{2}+4\|\Delta v\|^{2} .
$$

It can easily be shown that

$$
\begin{gathered}
\left|\left(2 \Delta z\left(\theta_{t} \omega\right), \Delta^{2} v\right)\right| \leq \frac{1}{12}\left\|\Delta^{2} v\right\|^{2}+C\left\|\Delta z\left(\theta_{t} \omega\right)\right\|^{2}, \\
\left|\left(g(x, t), \Delta^{2} v\right)\right| \leq \frac{1}{12}\left\|\Delta^{2} v\right\|^{2}+3\|g(t)\|^{2} .
\end{gathered}
$$

It is evident that

$$
\left|\left(\Delta^{2} z\left(\theta_{t} \omega\right), \Delta^{2} v\right)\right| \leq \frac{1}{12}\left\|\Delta^{2} v\right\|^{2}+C\left\|z\left(\theta_{t} \omega\right)\right\|^{2} .
$$

Since

$$
\begin{aligned}
& \|v\|_{L^{8}} \leq C\left\|\Delta^{2} v\right\|^{3 / 16}\|v\|^{13 / 16}, \\
& \|v\|_{L^{4}} \leq C\left\|\Delta^{2} v\right\|^{1 / 8}\|v\|^{7 / 8},
\end{aligned}
$$

we deduce that

$$
\begin{gathered}
\left|\left(5 v^{4} z\left(\theta_{t} \omega\right), \Delta^{2} v\right)\right| \leq 5\|v\|_{L^{8}}^{4}\left\|\Delta^{2} v\right\|\left\|z\left(\theta_{t} \omega\right)\right\|_{L^{\infty}} C \\
\left\|\Delta^{2} v\right\|^{7 / 4}\|v\|^{13 / 4}\left\|z\left(\theta_{t} \omega\right)\right\|_{L^{\infty}} \leq \frac{1}{12}\left\|\Delta^{2} v\right\|^{2} \\
+C\left\|z\left(\theta_{t} \omega\right)\right\|_{L^{\infty}}^{8}, \\
\left|\left(5 v z^{4}\left(\theta_{t} \omega\right), \Delta^{2} v\right)\right| \leq 5\|v\|_{L^{4}}\left\|\Delta^{2} v\right\|\left\|z\left(\theta_{t} \omega\right)\right\|_{L^{16}}^{4} \\
\leq C\left\|\Delta^{2} v\right\|^{9 / 8}\left\|z\left(\theta_{t} \omega\right)\right\|_{L^{16}}^{4} \leq \frac{1}{12}\left\|\Delta^{2} v\right\|^{2} \\
+C\left\|z\left(\theta_{t} \omega\right)\right\|_{L^{16}}^{64 / 7},
\end{gathered}
$$

where we have used the boundedness of $\|v\|$. It shows that

$$
\left|\left(z^{5}\left(\theta_{t} \omega\right), \Delta^{2} v\right)\right| \leq \frac{1}{12}\left\|\Delta^{2} v\right\|^{2}+C\left\|z\left(\theta_{t} \omega\right)\right\|_{L^{10}}^{10} .
$$

Similarly, we can get

$$
\begin{aligned}
& \left|\left(10 v^{3} z^{2}\left(\theta_{t} \omega\right), \Delta^{2} v\right)\right| \\
& \quad \leq 10\|v\|_{L^{12}}^{3}\left\|\Delta^{2} v\right\|\left\|z\left(\theta_{t} \omega\right)\right\|_{L^{8}}^{2} \\
& \leq C\left\|\Delta^{2} v\right\|^{13 / 8}\left\|z\left(\theta_{t} \omega\right)\right\|_{L^{8}}^{2}\|v\|^{19 / 8} \leq C\left\|\Delta^{2} v\right\|^{13 / 8}\left\|z\left(\theta_{t} \omega\right)\right\|_{L^{8}}^{2} \\
& \quad \leq \frac{1}{12}\left\|\Delta^{2} v\right\|^{2}+C\left\|z\left(\theta_{t} \omega\right)\right\|_{L^{8}}^{32 / 3}, \\
& \left(10 v^{2} z^{3}\left(\theta_{t} \omega\right), \Delta^{2} v\right) \mid \\
& \quad \leq 10\|v\|_{L^{8}}^{2}\left\|\Delta^{2} v\right\|\left\|z\left(\theta_{t} \omega\right)\right\|_{L^{12}}^{3} \\
& \quad \leq C\left\|\Delta^{2} v\right\|^{3 / 8}\|v\|^{13 / 8}\left\|\Delta^{2} v\right\|\left\|z\left(\theta_{t} \omega\right)\right\|_{L^{12}}^{3} \\
& \quad \leq C\left\|\Delta^{2} v\right\|^{11 / 8}\left\|z\left(\theta_{t} \omega\right)\right\|_{L^{12}}^{3} \leq \frac{1}{12}\left\|\Delta^{2} v\right\|^{2}+C\left\|z\left(\theta_{t} \omega\right)\right\|_{L^{12}}^{48 / 5} .
\end{aligned}
$$

Similarly, we have the following estimates:

$$
\int_{D}(\nabla v)^{2} v^{3}\left(\nabla^{2} v\right) \mathrm{d} x
$$

$$
\leq\|\nabla v\|_{L^{\infty}}^{2}\left\|\nabla^{2} v\right\|\|v\|_{L^{6}}^{3}
$$$$
\leq C\|v\|\left\|\Delta^{2} v\right\|\|\Delta v\|\|v\|_{L^{6}}^{3} \leq C\left\|\Delta^{2} v\right\|\|\Delta v\|\|v\|_{L^{6}}^{3}
$$

$$
\leq \frac{1}{240}\left\|\Delta^{2} v\right\|^{2}+C\|\Delta v\|^{2}\|v\|_{L^{6}}^{6},
$$

$$
\left(v^{5}, \Delta^{2} v\right)=-5 \int_{D} v^{4} \nabla v\left(\nabla^{3} v\right) \mathrm{d} x=5 \int_{D} \nabla\left(\nabla v v^{4}\right)\left(\nabla^{2} v\right) \mathrm{d} x
$$

$$
\begin{aligned}
& =5 \int_{D}\left(\nabla^{2} v v^{4}+4(\nabla v)^{2} v^{3}\right)\left(\nabla^{2} v\right) d x \\
& =5 \int_{D}\left(\nabla^{2} v\right)^{2} v^{4} d x+20 \int_{D}(\nabla v)^{2} v^{3}\left(\nabla^{2} v\right) \mathrm{d} x,
\end{aligned}
$$

where $\int_{D}\left(\nabla^{2} v\right)^{2} v^{4} \mathrm{~d} x \geq 0$.

Finally, we obtain

$\frac{\mathrm{d}}{\mathrm{d} t}\|\Delta v\|^{2}+(2 a-8)\|\Delta v\|^{2} \leq C\|v\|_{L^{6}}^{6}\|\Delta v\|^{2}+H\left(\theta_{t} \omega\right)+3\|g(t)\|^{2}$,

where

$$
\begin{aligned}
H\left(\theta_{t} \omega\right)= & C\left(\left|\widetilde{z}\left(\theta_{t} \omega\right)\right|^{2}+\left|\widetilde{z}\left(\theta_{t} \omega\right)\right|^{10}+\left|\widetilde{z}\left(\theta_{t} \omega\right)\right|^{32 / 3}\right) \\
& +\left|\widetilde{z}\left(\theta_{t} \omega\right)\right|^{8}+\left|\widetilde{z}\left(\theta_{t} \omega\right)\right|^{64 / 7}+\left|\widetilde{z}\left(\theta_{t} \omega\right)\right|^{48 / 5} .
\end{aligned}
$$

Therefore,

$$
\frac{\mathrm{d}}{\mathrm{d} t}\|\Delta v\|^{2} \leq C\|v\|_{L^{6}}^{6}\|\Delta v\|^{2}+H\left(\theta_{t} \omega\right)+3\|g(t)\|^{2} .
$$

For $s \in[\kappa-1, \kappa]$, choosing $t \geq T_{1}(\kappa, \omega, B) \geq 1$, by the Gronwall inequality on the interval $[s, \kappa]$, we obtain

$$
\begin{aligned}
& \left\|\Delta v\left(\kappa, \kappa-t, \theta_{-\kappa} \omega, v_{\kappa-t}\right)\right\|^{2} \\
& \leq e \int_{\kappa-1}^{\kappa} C\|v\|_{L^{6}}^{6} d \sigma\left(\left\|\Delta v\left(s, \kappa-t, \theta_{-\kappa} \omega, v_{\kappa-t}\right)\right\|^{2}\right. \\
& \quad+\int_{\kappa-1}^{\kappa} H\left(\theta_{r-\kappa} \omega\right) \mathrm{d} r \\
& \left.\quad+3 \int_{\kappa-1}^{\kappa}\|g(r)\|^{2} \mathrm{~d} r\right) .
\end{aligned}
$$

By Remark 1, one can obtain

$$
\int_{\kappa-1}^{\kappa}\left\|v\left(s, \kappa-t, \theta_{-\kappa} \omega, v_{\kappa-t}\right)\right\|_{L^{6}}^{6} \mathrm{~d} s \leq C r_{3}(\kappa, \omega) .
$$




$$
\begin{aligned}
& \left\|\Delta v\left(\kappa, \kappa-t, \theta_{-\kappa} \omega, v_{\kappa-t}\right)\right\|^{2} \leq e \int_{\kappa-1}^{\kappa} C\|v\|_{L^{6}}^{6} \mathrm{~d} \sigma \\
& \left(\int_{\kappa-1}^{\kappa}\left\|\Delta v\left(s, \kappa-t, \theta_{-\kappa} \omega, v_{\kappa-t}\right)\right\|^{2} \mathrm{~d} s+\int_{-1}^{0}\left(H\left(\theta_{r} \omega\right)\right.\right. \\
& \left.\left.+3\|g(r+\kappa)\|^{2}\right) \mathrm{~d} r\right) \leq e^{C r_{3}(\kappa, \omega)}\left(r_{2}(\kappa, \omega)+\frac{2 C}{a-6} r(\omega)\right. \\
& \left.+3 \int_{-1}^{0}\|g(r+\kappa)\|^{2} \mathrm{~d} r\right) .
\end{aligned}
$$

By Remark 1, (39)-(41), we can obtain

$$
\left\|\Delta v\left(\kappa, \kappa-t, \theta_{-\kappa} \omega, v_{\kappa-t}\right)\right\|^{2} \leq r_{4}(\kappa, \omega) .
$$

Furthermore,

$$
\left\|\Delta u\left(\kappa, \kappa-t, \theta_{-\kappa} \omega, u_{\kappa-t}\right)\right\|^{2} \leq r_{5}(\kappa, \omega) .
$$

Theorem 1. Suppose $a>6$ and (15) holds. There exists $a$ unique $\mathscr{D}$-pullback random attractor $\mathscr{A}=\{\mathscr{A}(\kappa, \omega): \kappa \in \mathbb{R}, \omega \in \Omega\} \in \mathscr{D}$ in $H=L^{2}(D)$ for continuous cocycle $\Phi$ of systems (1) and (2).

In the proof of the above lemmas, we find that all the estimates of solution do not depend on bounded domains D, so these estimates are also valid for unbounded domain.

Lemma 3. Suppose $a>6$ and (15) holds. Then, for every $\kappa \in \mathbb{R}, \omega \in \Omega$, and $B=\{B(\kappa, \omega): \kappa \in \mathbb{R}, \omega \in \Omega\} \in \mathscr{D}$, there are $T_{2}(\kappa, \omega, B, \epsilon) \geq T_{1} \geq 1$ and $k_{3}(\kappa, \omega, \epsilon) \geq 1$ satisfying

$$
\int_{|x| \geq k}\left|v\left(\kappa, \kappa-t, \theta_{-\kappa} \omega, v_{\kappa-t}\right)\right|^{2} \mathrm{~d} x \leq \epsilon, \forall t \geq T_{2}(\kappa, \omega, B, \epsilon), k \geq k_{3},
$$

where $v$ is the solution of systems (9)-(11) and $v_{\kappa-t}+z\left(\theta_{-t} \omega\right) \in B\left(\kappa-t, \theta_{-t} \omega\right)$.

Proof. Let $0 \leq \theta(s) \leq 1\left(s \in \mathbb{R}^{+}\right)$be a smooth function. When $0 \leq s \leq 1, \quad \theta(s)=0$. When $s \geq 2, \quad \theta(s)=1$. Then, $\left|\theta^{\prime}(s)\right|+\left|\theta^{\prime \prime}(s)\right|<C$. Multiplying (21) with $\theta\left(|x|^{2} / k^{2}\right) v$, we have

$$
\begin{aligned}
& \frac{1}{2} \frac{\mathrm{d}}{\mathrm{d} t} \int_{\mathbb{R}^{2}} \theta\left(\frac{|x|^{2}}{k^{2}}\right)|v|^{2} \mathrm{~d} x+\int_{\mathbb{R}^{2}} \theta\left(\frac{|x|^{2}}{k^{2}}\right) v\left(\Delta^{2} v+2 \Delta v\right) \mathrm{d} x \\
& \quad+\int_{\mathbb{R}^{2}} \theta\left(\frac{|x|^{2}}{k^{2}}\right) v\left(2 \Delta z\left(\theta_{t} \omega\right)+a v+v^{5}+\Delta^{2} z\left(\theta_{t} \omega\right)\right) \mathrm{d} x \\
& \quad+5 \int_{\mathbb{R}^{2}} \theta\left(\frac{|x|^{2}}{k^{2}}\right) v z\left(v^{4}+2 v^{3} z+2 v^{2} z^{2}+v z^{3}+\frac{1}{5} z^{4}\right) \mathrm{d} x \\
& \quad=\int_{\mathbb{R}^{2}} \theta\left(\frac{|x|^{2}}{k^{2}}\right) v g(x, t) \mathrm{d} x .
\end{aligned}
$$

For the estimate of $\int_{\mathbb{R}^{2}} \theta\left(|x|^{2} / k^{2}\right) v \Delta^{2} v \mathrm{~d} x$, similar to [21], it follows that

$$
\begin{aligned}
\int_{\mathbb{R}^{2}} \theta\left(\frac{|x|^{2}}{k^{2}}\right) v \Delta^{2} v \mathrm{~d} x \geq & -\frac{C}{k}\left(\|v\|^{2}+\|\nabla v\|^{2}+\|\Delta v\|^{2}\right) \\
& +\int_{\mathbb{R}^{2}} \theta\left(\frac{|x|^{2}}{k^{2}}\right)|\Delta v|^{2} \mathrm{~d} x .
\end{aligned}
$$

In addition, there are

$$
\begin{aligned}
\left|\int_{\mathbb{R}^{2}} \theta\left(\frac{|x|^{2}}{k^{2}}\right) v \Delta v \mathrm{~d} x\right| \leq & \frac{1}{2} \int_{\mathbb{R}^{2}} \theta\left(\frac{|x|^{2}}{k^{2}}\right)|\Delta v|^{2} \mathrm{~d} x+\frac{1}{2} \int_{\mathbb{R}^{2}} \theta\left(\frac{|x|^{2}}{k^{2}}\right)|v|^{2} \mathrm{~d} x, \\
& \left|\int_{\mathbb{R}^{2}} \theta\left(\frac{|x|^{2}}{k^{2}}\right) v \Delta^{2} z\left(\theta_{t} \omega\right) \mathrm{d} x\right| \leq \frac{a}{2} \int_{\mathbb{R}^{2}} \theta\left(\frac{|x|^{2}}{k^{2}}\right)|v|^{2} \mathrm{~d} x+C \int_{\mathbb{R}^{2}} \theta\left(\frac{|x|^{2}}{k^{2}}\right)\left|\Delta^{2} z\left(\theta_{t} \omega\right)\right|^{2} \mathrm{~d} x \\
& \cdot \int_{\mathbb{R}^{2}} \theta\left(\frac{|x|^{2}}{k^{2}}\right) v g(x, t) \mathrm{d} x \leq \frac{1}{2} \int_{\mathbb{R}^{2}} \theta\left(\frac{|x|^{2}}{k^{2}}\right)|v|^{2} \mathrm{~d} x+C \int_{\mathbb{R}^{2}} \theta\left(\frac{|x|^{2}}{k^{2}}\right)|g(t)|^{2} \mathrm{~d} x .
\end{aligned}
$$

Similarly, we get

$$
\begin{aligned}
& \left|\int_{\mathbb{R}^{2}} \theta\left(\frac{|x|^{2}}{k^{2}}\right) v \Delta z\left(\theta_{t} \omega\right) \mathrm{d} x\right| \leq \frac{1}{2} \int_{\mathbb{R}^{2}} \theta\left(\frac{|x|^{2}}{k^{2}}\right)|v|^{2} \mathrm{~d} x \\
& +C \int_{\mathbb{R}^{2}} \theta\left(\frac{|x|^{2}}{k^{2}}\right)\left|\Delta z\left(\theta_{t} \omega\right)\right|^{2} \mathrm{~d} x,
\end{aligned}
$$




$$
\begin{aligned}
\left|\int_{\mathbb{R}^{2}} \theta\left(\frac{|x|^{2}}{k^{2}}\right) v^{5} z\left(\theta_{t} \omega\right) \mathrm{d} x\right| & \leq \frac{1}{10} \int_{\mathbb{R}^{2}} \theta\left(\frac{|x|^{2}}{k^{2}}\right)|v|^{6} \mathrm{~d} x+C \int_{\mathbb{R}^{2}} \theta\left(\frac{|x|^{2}}{k^{2}}\right)\left|z\left(\theta_{t} \omega\right)\right|^{6} \mathrm{~d} x \\
& \left|\int_{\mathbb{R}^{2}} \theta\left(\frac{|x|^{2}}{k^{2}}\right) v z^{5}\left(\theta_{t} \omega\right) \mathrm{d} x\right| \leq \frac{1}{2} \int_{\mathbb{R}^{2}} \theta\left(\frac{|x|^{2}}{k^{2}}\right)|v|^{2} \mathrm{~d} x+C \int_{\mathbb{R}^{2}} \theta\left(\frac{|x|^{2}}{k^{2}}\right)\left|z\left(\theta_{t} \omega\right)\right|^{10} \mathrm{~d} x \\
& \left|\int_{\mathbb{R}^{2}} \theta\left(\frac{|x|^{2}}{k^{2}}\right) v^{3} z^{3}\left(\theta_{t} \omega\right) \mathrm{d} x\right| \leq \frac{1}{20} \int_{\mathbb{R}^{2}} \theta\left(\frac{|x|^{2}}{k^{2}}\right)|v|^{6} \mathrm{~d} x \\
& +C \int_{\mathbb{R}^{2}} \theta\left(\frac{|x|^{2}}{k^{2}}\right)\left|z\left(\theta_{t} \omega\right)\right|^{6} \mathrm{~d} x
\end{aligned}
$$

Because $\quad \int_{\mathbb{R}^{2}} \theta\left(|x|^{2} / k^{2}\right) v^{4} z^{2}\left(\theta_{t} \omega\right) \mathrm{d} x \geq 0 \quad$ and $\int_{\mathbb{R}^{2}} \theta\left(|x|^{2} / k^{2}\right) v^{2} z^{4}\left(\theta_{t} \omega\right) \mathrm{d} x \geq 0$, one yields

$$
\begin{aligned}
& \frac{\mathrm{d}}{\mathrm{d} t} \int_{\mathbb{R}^{2}} \theta\left(\frac{|x|^{2}}{k^{2}}\right)|v|^{2} \mathrm{~d} x+(a-6) \int_{\mathbb{R}^{2}} \theta\left(\frac{|x|^{2}}{k^{2}}\right)|v|^{2} \mathrm{~d} x \leq \frac{C}{k}\left(\|v\|^{2}+\|\nabla v\|^{2}+\|\Delta v\|^{2}\right) \\
& \quad+C \int_{\mathbb{R}^{2}} \theta\left(\frac{|x|^{2}}{k^{2}}\right)\left(|\Delta z|^{2}+|z|^{6}+\left|\Delta^{2} z\right|^{2}+|z|^{10}\right) \mathrm{d} x+C \int_{\mathbb{R}^{2}} \theta\left(\frac{|x|^{2}}{k^{2}}\right)|g(t)|^{2} \mathrm{~d} x .
\end{aligned}
$$

Applying the Gronwall lemma on $[\kappa-t, \kappa]$, one gets

$$
\begin{aligned}
\int_{\mathbb{R}^{2}} \theta\left(\frac{|x|^{2}}{k^{2}}\right)\left|v\left(\kappa, \kappa-t, \theta_{-\kappa} \omega, v_{\kappa-t}\right)\right|^{2} \mathrm{~d} x \leq & e^{-(a-6) t}\left\|v_{\kappa-t}\right\|^{2}+\frac{C}{k} \int_{-t}^{0} e^{(a-6) s}\left(\left\|v\left(s+\kappa, \kappa-t, \theta_{-\kappa} \omega, v_{\kappa-t}\right)\right\|^{2}\right. \\
& \left.+\left\|\nabla v\left(s+\kappa, \kappa-t, \theta_{-\kappa} \omega, v_{\kappa-t}\right)\right\|^{2}+\left\|\Delta v\left(s+\kappa, \kappa-t, \theta_{-\kappa} \omega, v_{\kappa-t}\right)\right\|^{2}\right) \mathrm{d} s \\
& +C \int_{-\infty}^{0} e^{(a-6) s} \int_{\mathbb{R}^{2}} \theta\left(\frac{|x|^{2}}{k^{2}}\right)\left(\left|\Delta z\left(\theta_{s} \omega\right)\right|^{2}+\left|\Delta^{2} z\left(\theta_{s} \omega\right)\right|^{2}+\left|z\left(\theta_{s} \omega\right)\right|^{6}+\left|z\left(\theta_{s} \omega\right)\right|^{10}\right) \mathrm{d} x \mathrm{~d} s \\
& +C \int_{-\infty}^{0} e^{(a-6) s} \int_{\mathbb{R}^{2}} \theta\left(\frac{|x|^{2}}{k^{2}}\right)|g(s+\kappa)|^{2} \mathrm{~d} x \mathrm{~d} s
\end{aligned}
$$

Noticing $v_{\kappa-t}+z\left(\theta_{-t} \omega\right) \in B\left(\kappa-t, \theta_{-t} \omega\right)$ and $B \in \mathscr{D}$, there is a $T_{2} \geq T_{1}(t, \omega, B, \epsilon)$. When $t \geq T_{2}$, we have

$$
e^{-(a-6) t}\left\|v_{\kappa-t}\right\|^{2} \leq \frac{\epsilon}{4}
$$

From (51), combining with Remark 1, if $\epsilon>0$, there is a $k_{1}(\kappa, \epsilon) \geq 1$ satisfying

$$
\frac{C}{k} \int_{-t}^{0} e^{(a-6) s}\left(\|v\|^{2}+\|\Delta v\|^{2}\right) \mathrm{d} s \leq \frac{\epsilon}{4}, \forall k \geq k_{1}(\kappa, \epsilon) .
$$

And there is a $k_{2}=k_{2}(\epsilon) \geq k_{1}(\kappa, \epsilon)$ such that

$$
\begin{aligned}
& C \int_{-\infty}^{0} e^{(a-6) s} \int_{\mathbb{R}^{2}} \theta\left(\frac{|x|^{2}}{k^{2}}\right)\left(\left|\Delta z\left(\theta_{s} \omega\right)\right|^{2}+\left|\Delta^{2} z\left(\theta_{s} \omega\right)\right|^{2}\right. \\
& \left.\quad+\left|z\left(\theta_{s} \omega\right)\right|^{6}+\left|z\left(\theta_{s} \omega\right)\right|^{10}\right) \mathrm{d} x \mathrm{~d} s \leq C \epsilon \int_{-\infty}^{0} \\
& \quad e^{(a-6) s}\left(\left|\widetilde{z}\left(\theta_{s} \omega\right)\right|^{2}+\left|\widetilde{z}\left(\theta_{s} \omega\right)\right|^{6}+\left|\widetilde{z}\left(\theta_{s} \omega\right)\right|^{10}\right) \mathrm{d} s \leq \frac{\epsilon}{4}, k \geq k_{2} .
\end{aligned}
$$

Meanwhile, there is a $k_{3}(\kappa, \epsilon) \geq k_{2}$ satisfying 


$$
C \int_{-\infty}^{0} e^{(a-6) s} \int_{\mathbb{R}^{2}} \theta\left(\frac{|x|^{2}}{k^{2}}\right)|g(s+\kappa)|^{2} \mathrm{~d} x \mathrm{~d} s \leq \frac{\epsilon}{4}, k \geq k_{3}(\kappa, \epsilon) .
$$

By (51)-(56), we deduce

$$
\int_{|x| \geq k}\left|v\left(\kappa, \kappa-t, \theta_{-\kappa} \omega, v_{\kappa-t}\right)\right|^{2} \mathrm{~d} x \leq \epsilon, \quad \forall k \geq k_{3}(\kappa, \epsilon) .
$$

Lemma 4. Suppose $a>6$ and (15) holds. Then, given $\epsilon>0$ and $B=\{B(, \omega): \kappa \in \mathbb{R}, \quad \omega \in \Omega\} \in \mathscr{D}, \quad \exists T_{2}=T_{2}(\kappa, \omega$, $B, \epsilon) \geq T_{1} \geq 1$, and $k_{4}=k_{4}(\omega, \epsilon)>0$, the solution $u\left(\kappa, \kappa-t, \theta_{\kappa} \omega, u_{\kappa-t}\right)=v\left(\kappa, \kappa-t, \theta_{\kappa} \omega, v_{\kappa-t}\right)+z\left(\theta_{t} \omega\right)$ satisfies the following inequality:

$$
\int_{|x| \geq k^{\prime}}\left|u\left(\kappa, \kappa-t, \theta_{-\kappa} \omega, u_{\kappa-t}\right)\right|^{2} \mathrm{~d} x \leq \epsilon, \quad \forall t \geq T_{2}, k^{\prime} \geq k_{4},
$$

where $v_{\kappa-t}+z\left(\theta_{-t} \omega\right) \in B\left(\kappa-t, \theta_{-t} \omega\right)$.

Proof. Since $z\left(\theta_{t} \omega\right) \in H^{4}\left(\mathbb{R}^{2}\right)$, then there exists a $k_{1}^{\prime}>0$ such that

$$
\int_{|x| \geq k_{1}^{\prime}}\left|z\left(\theta_{t} \omega\right)\right|^{2} \mathrm{~d} x \leq \epsilon\left|\widetilde{z}\left(\theta_{t} \omega\right)\right|^{2}
$$

By Lemma 3, $\exists k_{2}^{\prime}>k_{3}>0$, then

$$
\int_{|x| \geq k_{2}^{\prime}}\left|v\left(\kappa, \kappa-t, \theta_{-\kappa} \omega, v_{\kappa-t}\right)\right|^{2} \mathrm{~d} x \leq \frac{\epsilon}{4}, \quad \forall t \geq T_{2}(\kappa, \omega, B, \epsilon) .
$$
gets

By (55) and (56), $\forall t \geq T_{2}$ and $k^{\prime} \geq k_{4}=\max \left\{k_{1}^{\prime}, k_{2}^{\prime}\right\}$, one

$$
\int_{|x| \geq k^{\prime}}\left|u\left(\kappa, \kappa-t, \theta_{-\kappa} \omega, u_{\kappa-t}\right)\right|^{2} \mathrm{~d} x \leq \epsilon .
$$

\section{Finiteness of Fractal Dimension}

Now, we are devoted to the existence of random attractor on $\mathbb{R}^{2}$ for the random dynamical system $\Phi$.

Lemma 5. Suppose $a>6$ and (15) holds. Then, D-pullback asymptotically compact holds in $L^{2}\left(\mathbb{R}^{2}\right)$ for continuous cocycle $\Phi$ of (1) and (2).

Similar to the method in [30], we only give the sketch of the proof for Lemma 5.

Firstly, the weak convergence

$$
\Phi\left(t_{n}, \kappa-t_{n}, \theta_{-t_{n}} \omega, u_{0, n}\right)-\xi
$$

can be given in $L^{2}\left(\mathbb{R}^{2}\right)$.

Secondly, by Lemma 4 , there exist enough large $t$ and $M$ satisfying

$$
\int_{|x| \geq M}\left|\Phi\left(t, \kappa-t, \theta_{-t} \omega, u_{0}\right)\right|^{2 \mathrm{~d} x \leq \epsilon}
$$

Denote the set $Q_{M}=\left\{x \in \mathbb{R}^{2}:|x| \leq M\right\}$. By the estimates of Lemma 2, the embedding $H^{2}\left(Q_{M}\right) \mapsto L^{2}\left(Q_{M}\right)$ is compact. It follows that the strong convergence

$$
\Phi\left(t_{n}, \kappa-t_{n}, \theta_{-t_{n}} \omega, u_{0, n}\right) \longrightarrow \xi
$$

holds in $L^{2}\left(\mathbb{R}^{2}\right)$

According to [30], the following theorem is easily obtained. The proof is omitted.

Theorem 2. Suppose $a>6$ and (15) holds. Then, the continuous cocycle $\Phi$ corresponding to problems (1) and (2) has a unique $\mathscr{D}$-pullback attractor $\mathscr{A}=\{\mathscr{A}(\kappa, \omega)$ : $\kappa \in \mathbb{R}, \omega \in \Omega\} \in \mathscr{D}$ in $L^{2}\left(\mathbb{R}^{2}\right)$.

By Theorem 1, similar to the continuous cocycle $\Phi$ defined in (12), the random dynamical system $\Psi$ defined in (13) has a unique $\mathscr{D}$-pullback attractor, denoted by $\tilde{\mathscr{A}}=$ $\{\tilde{\mathscr{A}}(\kappa, \omega): \kappa \in \mathbb{R}, \omega \in \Omega\}$ in $H=L^{2}(D) \in \mathscr{D}$. The boundedness of fractal dimension is proved for the random dynamical system $\Psi$. Because of complexity of proof on unbounded domain, we pay attention to studying the case of bounded domain $D$. Especially, the space $L^{2}(D)$ is denoted by $H$.

Assume that $\{\chi(\kappa, \omega)\}_{\kappa \in \mathbb{R}, \omega \in \Omega}$ of $X$ are a family of bounded closed random subsets, which satisfy the below conditions. For $\forall \omega \in \Omega, \kappa \in \mathbb{R}$, the following holds:

$H 1$ : there is tempered random variable $R_{\omega}$, which is not dependent on $\kappa$ and satisfies the diameter $\|\chi(\kappa, \omega)\|_{X}$ of $\chi(\kappa, \omega)$ controlled by $R_{\omega}$, and $R_{\theta_{t} \omega}(\forall t \in \mathbb{R})$ is continuous.

$H 2$ : for $\forall t>0, \chi\left(t+\kappa, \theta_{t} \omega\right)=\Psi(t, \kappa, \omega) \chi(\kappa, \omega)$.

H3: there are random variables $C_{0}(\omega) \geq 0$, positive numbers $\lambda, \delta, t_{0}$, and projector $P_{m}: X \longrightarrow P_{m} X$ satisfying

$$
\begin{aligned}
& \left\|P_{m} \Psi\left(t_{0}, \kappa, \omega\right) v-P_{m} \Psi\left(t_{0}, \kappa, \omega\right) u\right\|_{X} \leq e^{\int_{0}^{t_{0}} C_{0}\left(\theta_{s} \omega\right) \mathrm{d} s}\|v-u\|_{X} \\
& \left\|\left(I-P_{m}\right) \Psi\left(t_{0}, \kappa, \omega\right) v-\left(I-P_{m}\right) \Psi\left(t_{0}, \kappa, \omega\right) u\right\|_{X} \\
& \quad \leq\left(e^{-\lambda t_{0}}+\delta e \int_{0}^{t_{0}} C_{0}\left(\theta_{s} \omega\right) \mathrm{d} s\right)\|v-u\|_{X}
\end{aligned}
$$

for any $u, v \in \chi(\kappa, \omega), \quad \kappa \in \mathbb{R}$, and $\omega \in \Omega$, where $\lambda, \delta, t_{0}, m$ do not depend on $\kappa, \omega$.

$H 4: \lambda, \delta, t_{0}, C_{0}(\omega)$ satisfy the following conditions: 


$$
\left\{\begin{array}{l}
0 \leq E\left[C_{0}(\omega)\right]<\infty \\
\lambda \geq 32 E\left[C_{0}(\omega)\right] \\
0<\delta \leq \frac{1}{32} \\
4 \ln 2 \leq \lambda t_{0} \leq 8 \ln 2
\end{array}\right.
$$

where " $E$ " denotes the expectation of random variables.

Once these conditions $(H 1)-(H 4)$ are satisfied, the finiteness of fractal dimension can be obtained by further calculation $[18,25]$. We know that conditions $(H 1)$ and (H2) hold.

Lemma 6. Let $\omega \in \Omega, \kappa \in \mathbb{R}, t \geq 0$. We have

$$
\begin{aligned}
& \left\|\varphi_{1}\left(t+\kappa, \kappa, \theta_{-\kappa} \omega, \varphi_{1 \kappa}\left(\theta_{-\kappa} \omega\right)\right)-\varphi_{2}\left(t+\kappa, \kappa, \theta_{-\kappa} \omega, \varphi_{2 \kappa}\left(\theta_{-\kappa} \omega\right)\right)\right\| \\
& \leq e^{\int_{0}^{t}(1-a) \mathrm{d} s}\left\|\varphi_{1 \kappa}-\varphi_{2 \kappa}\right\|, \varphi_{1 \kappa}, \varphi_{2 \kappa} \in \tilde{\mathscr{A}} .
\end{aligned}
$$

Proof. Let $\varphi_{j}(r)=\varphi_{j}\left(r, \kappa, \omega, \varphi_{j \kappa}(\omega)\right), y(r)=\varphi_{1}(r)-\varphi_{2}(r)$, $j=1,2$, when $r \geq \kappa$. By equation (9), we have

$$
\left\{\begin{array}{l}
\frac{\mathrm{d} y}{\mathrm{~d} t}+\Delta^{2} y+2 \Delta y+a y+f\left(\varphi_{1}\right)-f\left(\varphi_{2}\right)=0, r \geq \kappa \\
y(r, \omega, x)=0, x \in \partial D \\
y(\kappa, \omega, x)=y_{\kappa}(\omega, x), \kappa \in \mathbb{R}, x \in D
\end{array}\right.
$$

where $f(\zeta)=(\zeta+z)^{5}$. Multiplying equation (69) with $y(r)$, noticing that

$$
\int_{D} y\left(f\left(\varphi_{1}\right)-f\left(\varphi_{2}\right)\right) \mathrm{d} x \geq 0
$$

we have

$$
\frac{\mathrm{d}}{\mathrm{d} r}\|y(r)\|^{2} \leq 2(1-a)\|y(r)\|^{2}, \forall r \geq \kappa .
$$

By the Gronwall inequality, we obtain the estimate (68). Let $\left\{e_{j}\right\}_{j \in \mathbb{N}}$ be the eigenvectors of operator $-\Delta$ corresponding to the eigenvalues $\left\{\lambda_{j}\right\}_{j \in \mathbb{N}}$ with $-\Delta e_{j}=\lambda_{j} e_{j}$ for $j \in \mathbb{N}$. Let $H_{n}$ be the space spanned by $\left\{e_{j}\right\}_{j=1}^{n}$ and $P_{n}: H \longrightarrow H_{n}$ be the orthonormal projector. Let $Q_{n}: H \longrightarrow H_{n}^{\perp}$ be the defined by $P_{n} v=v_{n}$ for $v \in H$, with $v=v_{n}+v_{n}^{\perp}$, where $v_{n} \in H_{n}, v_{n}^{\perp} \in H_{n}^{\perp}$. Then,

$$
\lambda_{n+1}\left\|Q_{n} v\right\|^{2} \leq\|v\|_{1}^{2} \text {. }
$$

Lemma 7. For $\varphi_{1 \kappa}, \varphi_{2 \kappa} \in \tilde{\mathscr{A}}(\kappa, \omega)$, there are a random variable $C_{0}(\omega) \geq 0$ and a projector $P_{n}: H \longrightarrow H_{n}$ satisfying

$$
\begin{aligned}
& \|\left(I-P_{n}\right) \Psi\left(t, \kappa, \theta_{-\kappa} \omega\right) \varphi_{1 \kappa}\left(\theta_{-\kappa} \omega\right)-\left(I-P_{n}\right) \\
& \Psi\left(t, \kappa, \theta_{-\kappa} \omega\right) \varphi_{2 \kappa}\left(\theta_{-\kappa} \omega\right) \|, \\
& \quad \leq\left(e^{\left(4-a-1 / 4 \lambda_{n+1}^{2}\right)^{t}}+\delta_{n} e^{\int_{0}^{t} C_{0}\left(\theta_{s} \omega\right) \mathrm{d} s}\right)\left\|\varphi_{1 \kappa}-\varphi_{2 \kappa}\right\|,
\end{aligned}
$$

and

$$
\begin{gathered}
\left\|P_{n} \Psi\left(t, \kappa, \theta_{-\kappa} \omega\right) \varphi_{1 \kappa}\left(\theta_{-\kappa} \omega\right)-P_{n} \Psi\left(t, \kappa, \theta_{-\kappa} \omega\right) \varphi_{2 \kappa}\left(\theta_{-\kappa} \omega\right)\right\| \\
\leq e^{\int_{0}^{t} C_{0}\left(\theta_{s} \omega\right) \mathrm{d} s}\left\|\varphi_{1 \kappa}-\varphi_{2 \kappa}\right\|, \quad \forall \kappa \in \mathbb{R}, \omega \in \Omega, t \geq 0
\end{gathered}
$$

where

$$
\delta_{n}=\frac{1}{\sqrt{\lambda_{n+1}}} .
$$

Proof. Multiplying equation (69) with $y_{n}=Q_{n} y$ in $H$, it follows that

$$
\left|\left(2 \Delta y, y_{n}\right)\right| \leq \frac{1}{4}\|\Delta y\|^{2}+4\left\|y_{n}\right\|^{2}
$$

$\|\nabla v\|^{2} \leq C\|\Delta v\|\|v\| \leq r_{1}(\omega)+C r_{8}(\omega)=M_{0}^{2}\left(\theta_{r} \omega\right)$.

Then, there exists a $\vartheta$ between $\varphi_{1}(r)$ and $\varphi_{2}(r)$ such that

$$
\begin{aligned}
\left(f\left(\varphi_{1}\right)-f\left(\varphi_{2}\right), y_{n}\right) \leq & 5\left(\int_{D}\left(\vartheta+z\left(\theta_{r} \omega\right)\right)^{12} \mathrm{~d} x\right)^{1 / 3} \\
& \cdot\left(\int_{D} y^{2} \mathrm{~d} x\right)^{1 / 2}\left(\int_{D} y_{n}^{6} \mathrm{~d} x\right)^{1 / 6} \\
& \leq C\left(\|\vartheta\|_{H_{0}^{1}}^{4}+\left\|z\left(\theta_{r} \omega\right)\right\|_{L^{12}}^{4}\right)\|y\|\left\|y_{n}\right\|_{L^{6}} \\
& \leq C\left(M_{0}^{4}\left(\theta_{r} \omega\right)+\left\|z\left(\theta_{r} \omega\right)\right\|_{L^{12}}^{4}\right)^{2}\|y\|^{2} \\
& +\frac{1}{2}\left\|\Delta y_{n}\right\|^{2}
\end{aligned}
$$

and

$\frac{\mathrm{d}}{\mathrm{d} t}\left\|y_{n}\right\|^{2}+\left(\frac{1}{2} \lambda_{n+1}^{2}+2 a-8\right)\left\|y_{n}\right\|^{2} \leq C\left(M_{0}^{4}\left(\theta_{r} \omega\right)+\left\|z\left(\theta_{r} \omega\right)\right\|_{L^{12}}^{4}\right)^{2}\|y\|^{2}$.

Applying Gronwall lemma to (78) and combining (68), one gets 


$$
\begin{aligned}
& \left\|y_{n}\left(\kappa+t, \kappa, \theta_{-\kappa} \omega, y_{\kappa}\left(\theta_{-\kappa} \omega\right)\right)\right\|^{2} \leq\left\|y_{n}(\kappa)\right\|^{2} e^{\int_{\kappa}^{\kappa+t}\left(8-2 a-1 / 2 \lambda_{n+1}^{2}\right) \mathrm{d} s}+\int_{\kappa}^{\kappa+t} C\left(M_{0}^{4}\left(\theta_{r-\kappa} \omega\right)+\left\|z\left(\theta_{r-\kappa} \omega\right)\right\|_{L^{2}}^{4}\right)^{2}\left\|\varphi_{1}(r)-\varphi_{2}(r)\right\|^{2} \\
& e^{\int_{r}^{\kappa+t}\left(8-2 a-1 / 2 \lambda_{n+1}^{2}\right) \mathrm{d} s} \mathrm{~d} r \leq\left\|y_{n}(\kappa)\right\|^{2} e^{\int_{0}^{t}\left(8-2 a-1 / 2 \lambda_{n+1}^{2}\right) \mathrm{d} s} \\
& +\left\|\varphi_{1 \kappa}-\varphi_{2 \kappa}\right\|^{2} \int_{\kappa}^{\kappa+t} C\left(M_{0}^{4}\left(\theta_{r-\kappa} \omega\right)+\left\|z\left(\theta_{r-\kappa} \omega\right)\right\|_{L^{12}}^{4}\right)^{2} e^{\int_{\kappa}^{r} 2(1-a) d s} e^{\int_{r}^{\kappa+t}\left(8-2 a-1 / 2 \lambda_{n+1}^{2}\right) \mathrm{d} s} \mathrm{~d} r \leq\left\|y_{n}(\kappa)\right\|^{2} \\
& e^{\int_{0}^{t}\left(8-2 a-1 / 2 \lambda_{n+1}^{2}\right) \mathrm{d} s}+\left\|\varphi_{1 \kappa}-\varphi_{2 \kappa}\right\|^{2} e^{\int_{0}^{t}(8-2 a) \mathrm{d} s} \int_{0}^{t} C\left(M_{0}^{4}\left(\theta_{r-\kappa} \omega\right)+\left\|z\left(\theta_{r-\kappa} \omega\right)\right\|_{L^{12}}^{4}\right)^{2} e^{-1 / 2 \lambda_{n+1}^{2}(t-r)} \mathrm{d} r .
\end{aligned}
$$

Noticing $\sqrt{x}<e^{x}(x \geq 0)$, we have

$$
\begin{aligned}
& \int_{0}^{t} C\left(M_{0}^{4}\left(\theta_{r} \omega\right)+\left\|z\left(\theta_{r} \omega\right)\right\|_{L^{12}}^{4}\right)^{2} \cdot e^{-1 / 2 \lambda_{n+1}^{2}(t-r)} \mathrm{d} r \leq\left(\int_{0}^{t} C^{2} \cdot\left(M_{0}^{4}\left(\theta_{r} \omega\right)+\left\|z\left(\theta_{r} \omega\right)\right\|_{L^{12}}^{4}\right)^{4} \mathrm{~d} r\right)^{\frac{1}{2}} \\
& \left(\int_{0}^{t} e^{-\lambda_{n+1}^{2}(t-r)} \mathrm{d} r\right)^{1 / 2} \leq \frac{1}{\lambda_{n+1}} e_{0}^{t} C_{0}^{2}\left(M_{0}^{4}\left(\theta_{r} \omega\right)+\left\|z\left(\theta_{r} \omega\right)\right\|_{L^{12}}^{4}\right)^{4} \mathrm{~d} r .
\end{aligned}
$$

By (79),

$$
\left\|y_{n}\left(\kappa+t, \kappa, \theta_{-\kappa} \omega, y_{\kappa}\left(\theta_{-\kappa} \omega\right)\right)\right\|^{2} \leq\left(e^{\int_{0}^{t}\left(8-2 a-1 / 2 \lambda_{n+1}^{2}\right) \mathrm{d} s}+\frac{1}{\lambda_{n+1}} e_{0}^{t}\left(8-2 a+C^{2}\left(M_{0}^{4}\left(\theta_{s} \omega\right)+\left\|z\left(\theta_{s} \omega\right)\right\|_{L 12}^{4}\right)^{4}\right) \mathrm{d} s\right)\left\|\varphi_{1 \kappa}-\varphi_{2 \kappa}\right\|^{2} /
$$

Thus,

$$
\left\|y_{n}\left(\kappa+t, \kappa, \theta_{-\kappa} \omega, y_{\kappa}\left(\theta_{-\kappa} \omega\right)\right)\right\| \leq\left(e^{\int_{0}^{t}\left(4-a-1 / 4 \lambda_{n+1}^{2}\right) d s}+\frac{1}{\sqrt{\lambda_{n+1}}} e^{\int_{0}^{t}\left(4-a+C^{2} / 2\left(M_{0}^{4}\left(\theta_{s} \omega\right)+\left\|z\left(\theta_{s} \omega\right)\right\|_{L^{12}}^{4}\right)^{4}\right) \mathrm{d} s}\right)\left\|\varphi_{1 \kappa}-\varphi_{2 \kappa}\right\|
$$

where $C_{0}(\omega)=4-a+C^{2} / 2\left(M_{0}^{4}(\omega)+\|z(\omega)\|_{L^{12}}^{4}\right)^{4}$. Then, (73) holds. From (68), it follows that (74) holds.

Lemma 8. The expectations of $C_{0}(\omega), C_{0}^{2}(\omega)$ are finite, that is,

$$
0 \leq E\left[C_{0}(\omega)\right], E\left[C_{0}^{2}(\omega)\right]<\infty
$$

Proof. Notice that $\tilde{z}\left(\theta_{t} \omega\right)$ satisfies $E\left[\left|\widetilde{z}\left(\theta_{t} \omega\right)\right|^{r}\right]=$ $\Gamma(1+r / 2) / \sqrt{\pi}$, for all $r>0, t \in \mathbb{R}$, and $\Gamma$ satisfies the equality [25]:

$$
\begin{gathered}
\Gamma\left(\frac{1+2 k}{2}\right)=\frac{(2 k-1)(2 k-3) \cdots 3 \cdot 1}{2^{k}} \\
\sqrt{\pi}, \Gamma\left(\frac{1+(2 k+1)}{2}\right)=k !, \forall k \in \mathbb{N} .
\end{gathered}
$$

By $M_{0}^{2}(\omega)=r_{1}(\omega)+C \cdot r_{8}(\omega)$,

$$
\begin{aligned}
& C_{0}(\omega)=\bar{C}\left(1+r_{1}^{8}(\omega)+r_{8}^{8}(\omega)+\left\|z\left(\theta_{t} \omega\right)\right\|_{L^{12}}^{16}\right) \\
& C_{0}^{2}(\omega)=C_{1}+C_{2} r_{1}^{16}(\omega)+C_{3} r_{8}^{16}(\omega)+C_{4}\left\|z\left(\theta_{t} \omega\right)\right\|_{L^{12}}^{32}
\end{aligned}
$$

where $\bar{C}, C_{1}, C_{2}, C_{3}, C_{4}$ are positive constants.

Since $r_{1}(\omega), r_{8}(\omega)$ are tempered, we obtain $E\left[r_{1}^{8}(\omega)\right]<\infty, \quad E\left[r_{8}^{8}(\omega)\right]<\infty \quad$ and $E\left[r_{1}^{16}(\omega)\right]<\infty$, $E\left[r_{8}^{16}(\omega)\right]<\infty$. By the properties of $\widetilde{z}\left(\theta_{t} \omega\right)$, we get

$$
\begin{aligned}
E\left[\left\|z\left(\theta_{t} \omega\right)\right\|_{L^{12}}^{16}\right]= & \|\phi(x)\|_{L^{12}}^{16} \cdot E\left[\left|\tilde{z}\left(\theta_{t} \omega\right)\right|^{16}\right] \\
& =\|\phi(x)\|_{L^{12}}^{16} \frac{15 \cdot 13 \cdot 11 \cdots 3 \cdot 1}{2^{8}}<\infty, \\
E\left[\left\|z\left(\theta_{t} \omega\right)\right\|_{L^{12}}^{32}\right]= & \|\phi(x)\|_{L^{12}}^{32} \cdot E\left[\left|\tilde{z}\left(\theta_{t} \omega\right)\right|^{32}\right] \\
& =\|\phi(x)\|_{L^{12}}^{32} \frac{31 \cdot 29 \cdot 27 \cdots 3 \cdot 1}{2^{16}}<\infty .
\end{aligned}
$$


Then,

$$
\begin{aligned}
E\left[C_{0}(\omega)\right]= & \bar{C}\left(1+E\left[r_{1}^{8}(\omega)\right]+E\left[r_{8}^{8}(\omega)\right]\right. \\
& \left.+E\left[\left\|z\left(\theta_{t} \omega\right)\right\|_{L^{12}}^{16}\right]\right)<\infty, \\
E\left[C_{0}^{2}(\omega)\right]= & C_{1}+C_{2} E\left[r_{1}^{16}(\omega)\right]+C_{3} E\left[r_{8}^{16}(\omega)\right] \\
& +C_{4} E\left[\left\|z\left(\theta_{t} \omega\right)\right\|_{L^{12}}^{32}\right]<\infty .
\end{aligned}
$$

Now, based on the conclusions of Lemmas 7 and 8, the main result is presented.

Theorem 3. The finiteness of fractal dimension for $\tilde{\mathscr{A}}(\kappa, \omega)$ holds, that is,

$$
\operatorname{dim}_{f} \tilde{\mathscr{A}}(\kappa, \omega) \leq \frac{8 n_{0} \ln \left(\sqrt{n_{0}} \lambda_{n_{0}+1}^{1 / 2}+1\right)}{\ln 2}<\infty, \kappa \in \mathbb{R}, \omega \in \Omega,
$$

where $\quad n_{0}=\min \left\{n: \lambda_{n+1} \geq \max \left\{\left(128 E \quad\left[C_{0}(\omega)\right]+16-\right.\right.\right.$ $\left.\left.4 a)^{1 / 2}, 1024\right\}\right\}<\infty$.

Proof. Comparing (66) and (73), we see that

$$
\lambda=\frac{1}{4} \lambda_{n+1}^{2}+a-4 \longrightarrow \infty, \delta=\frac{1}{\sqrt{\lambda_{n+1}}} \longrightarrow 0, \text { as } n \longrightarrow \infty \text {. }
$$

By (67) and Lemma $8, \exists n_{0} \in \mathbb{N}$, we deduce

$$
\frac{1}{4} \lambda_{n+1}^{2}+a-4 \geq 32 E\left[C_{0}(\omega)\right], 0<\frac{1}{\sqrt{\lambda_{n+1}}}<\frac{1}{32} .
$$

Take $t=t_{0}>0$ in (73) and (74) such that the following inequality is satisfied:

$$
0<\frac{16 \ln 2}{\lambda_{n+1}^{2}+4 a-16} \leq t_{0} \leq \frac{32 \ln 2}{\lambda_{n+1}^{2}+4 a-16}<\infty .
$$

If $(H 1)-(H 4)$ holds, the following estimate is similar to [25]; here, we omit the details. Then,

$$
\begin{aligned}
& \operatorname{dim}_{f} \tilde{\mathcal{A}}(\kappa, \omega) \leq \frac{8 n_{0} \ln \left(\sqrt{n_{0}} / \delta+1\right)}{\ln 2}=\frac{8 n_{0} \ln \left(\sqrt{n_{0}} \lambda_{n_{0}+1}^{1 / 2}+1\right)}{\ln 2} \\
& <\infty, \forall \kappa \in \mathbb{R}, \omega \in \Omega .
\end{aligned}
$$

\section{Data Availability}

The raw data supporting the conclusions of this article will be made available by the authors, without undue reservation, to any qualified researcher.

\section{Conflicts of Interest}

The authors declare that there are no conflicts of interest regarding the publication of this paper.

\section{Acknowledgments}

The paper was supported by the National Natural Science Foundation of China (nos. 11771444 and 11861013), Guangxi Natural Science Foundation (no. 2017GXNSFAA198221), Promotion of the Basic Capacity of Middle and Young Teachers in Guangxi Universities (no. 2017KY0340), and Fundamental Research Funds for the Central Universities, China University of Geosciences (Wuhan) (no. 2018061)

\section{References}

[1] J. Swift and P. C. Hohenberg, "Hydrodynamic fluctuations at the convective instability," Physical Review A, vol. 15, no. 1, pp. 319-328, 1977.

[2] D. Blömker, M. Hairer, and G. A. Pavliotis, "Stochastic SwiftHohenberg equation near a change of stability,"vol. 11, pp. 27-37, in Proceedings of the International Conference on Differential Equations, vol. 11, Comenius University, Bratislava, Slovakia, July 2005.

[3] M. F. Hilali, S. Métens, P. Borckmans, and G. Dewel, "Pattern selection in the generalized Swift-Hohenberg model," Physical Review E, vol. 51, no. 3, pp. 2046-2052, 1995.

[4] G. Lin, H. Gao, J. Duan, and V. J. Ervin, "Asymptotic dynamical difference between the nonlocal and local SwiftHohenberg models," Journal of Mathematical Physics, vol. 41, no. 4, pp. 2077-2089, 2000.

[5] J. Lega, J. V. Moloney, and A. C. Newell, "Swift-Hohenberg equation for lasers," Physical Review Letters, vol. 73, no. 22, pp. 2978-2981, 1994.

[6] L. Song, Y. Zhang, and T. Ma, "Global attractor of a modified Swift-Hohenberg equation in spaces," Nonlinear Analysis: Theory, Methods \& Applications, vol. 72, no. 1, pp. 183-191, 2010.

[7] L. A. Peletier and V. Rottschäfer, "Pattern selection of solutions of the Swift-Hohenberg equation," Physica D: Nonlinear Phenomena, vol. 194, no. 1-2, pp. 95-126, 2004.

[8] L. A. Peletier and V. Rottschäfer, "Large time behaviour of solutions of the Swift-Hohenberg equation," Comptes Rendus Mathematique, vol. 336, no. 3, pp. 225-230, 2003.

[9] L. A. Peletier and J. F. Williams, "Some canonical bifurcations in the Swift-Hohenberg equation," SIAM Journal on Applied Dynamical Systems, vol. 6, no. 1, pp. 208-235, 2007.

[10] S. H. Park and J. Y. Park, "Pullback attractor for a non-autonomous modified swift-hohenberg equation," Computers \& Mathematics with Applications, vol. 67, no. 3, pp. 542-548, 2014.

[11] Z. Wang and X. Du, "Pullback attractors for modified SwiftHohenberg equation on unbounded domains with non-autonomous deterministic and stochastic forcing terms," Journal of Applied Analysis and Computation, vol. 7, pp. 207-223, 2017.

[12] L. Xu and Q. Ma, "Existence of the uniform attractors for a non-autonomous modified Swift-Hohenberg equation," Advances in Differential Equations, vol. 2015, pp. 1-11, 2015.

[13] J. C. Robinson, Infinite-Dimensional Dynamical Systems, Cambridge University Press, Cambridge, UK, 2001.

[14] R. Temam, Infinite-Dimensional Dynamical Systems in $\mathrm{Me}$ chanics and Physics, Springer-Verlag, New York, NY, USA, 2nd edition, 1997. 
[15] C. Foias and E. J. Olson, "Finite fractal dimension and HölderLipschitz parameterization," Indiana University Journal, vol. 45, pp. 603-616, 1996.

[16] J. A. Langa and J. C. Robinson, "Fractal dimension of a random invariant set," Journal de Mathématiques Pures et Appliquées, vol. 85, no. 2, pp. 269-294, 2006.

[17] G. Wang and Y. Tang, "Fractal dimension of a random invariant set and applications," Journal of Applied Mathematics, vol. 2013, Article ID 415764, , 2013.

[18] A. Debussche, "On the finite dimensionality of random attractors," Stochastic Analysis and Applications, vol. 15, no. 4, pp. 473-491, 1997.

[19] J. A. Langa, "Finite-dimensional limiting dynamics of random dynamical systems," Dynamical Systems, vol. 18, no. 1, pp. 57-68, 2003.

[20] Y. Guo, "Dynamics and invariant manifolds for a nonlocal stochastic Swift-Hohenberg equation," Journal of Inequalities and Applications, vol. 2015, no. 366, 2015.

[21] C. Guo, Y. Chen, and Y. Guo, "Random attractors of stochastic local Swift-Hohenberg equation with additive noise," Journal of Inequalities and Applications, vol. 2016, no. 228, 2016.

[22] P. W. Bates, K. Lu, and B. Wang, "Random attractors for stochastic reaction-diffusion equations on unbounded domains," Journal of Differential Equations, vol. 246, no. 2, pp. 845-869, 2009.

[23] H. Crauel, A. Debussche, and F. Flandoli, "Random attractors," Journal of Dynamics and Differential Equations, vol. 9, no. 2, pp. 307-341, 1997.

[24] H. Crauel and F. Flandoli, "Attractors for random dynamical systems," Probability Theory and Related Fields, vol. 100, no. 3, pp. 365-393, 1994.

[25] S. Zhou, Y. Tian, and Z. Wang, "Fractal dimension of random attractors for stochastic non-autonomous reaction-diffusion equations," Applied Mathematics and Computation, vol. 276, pp. 80-95, 2016.

[26] S. Zhou and Z. Wang, "Finite fractal dimensions of random attractors for stochastic FitzHugh-Nagumo system with multiplicative white noise," Journal of Mathematical Analysis and Applications, vol. 441, no. 2, pp. 648-667, 2016.

[27] M. Polat, "Global attractor for a modified Swift-Hohenberg equation," Computers \& Mathematics with Applications, vol. 57, no. 1, pp. 62-66, 2009.

[28] B. Wang, "Asymptotic behavior of non-autonomous fractional stochastic reaction-diffusion equations," Nonlinear Analysis, vol. 158, pp. 60-82, 2017.

[29] L. Arnold, Random Dynamical Systems, Springer-Verlag, New York, NY, USA, 1998.

[30] B. Wang, "Random attractors for the stochastic FitzHughNagumo system on unbounded domains," Nonlinear Analysis, vol. 71, no. 7-8, pp. 2811-2828, 2009.

[31] M. Marion, "Finite-dimensional attractors associated with partly dissipative reaction-diffusion systems," SIAM Journal on Mathematical Analysis, vol. 20, no. 4, pp. 816-844, 1989. 